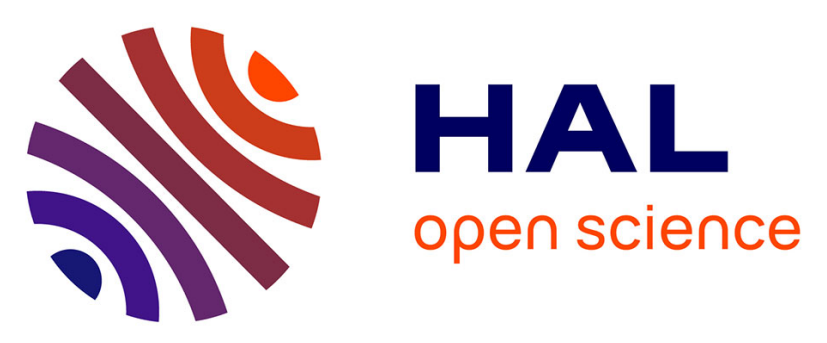

\title{
A new method of dielectric characterization in the microwave range for high-k ferroelectric thin films
}

Kevin Nadaud, Hartmut W. Gundel, Caroline Borderon, Raphaël Gillard, Erwan Fourn

\section{- To cite this version:}

Kevin Nadaud, Hartmut W. Gundel, Caroline Borderon, Raphaël Gillard, Erwan Fourn. A new method of dielectric characterization in the microwave range for high-k ferroelectric thin films. International Symposium on the Applications of Ferroelectrics, Jul 2013, Prague, Czech Republic. pp.9-12, 10.1109/ISAF.2013.6748698 . hal-00921681

\section{HAL Id: hal-00921681 \\ https://hal.science/hal-00921681}

Submitted on 13 Jan 2015

HAL is a multi-disciplinary open access archive for the deposit and dissemination of scientific research documents, whether they are published or not. The documents may come from teaching and research institutions in France or abroad, or from public or private research centers.
L'archive ouverte pluridisciplinaire HAL, est destinée au dépôt et à la diffusion de documents scientifiques de niveau recherche, publiés ou non, émanant des établissements d'enseignement et de recherche français ou étrangers, des laboratoires publics ou privés. 


\section{A new method of dielectric characterization in the microwave range for high-k ferroelectric thin films}

\author{
Kevin Nadaud, Hartmut W. Gundel \\ and Caroline Borderon \\ Institut d'Électronique et de Télécommunications de Rennes \\ University of Nantes \\ Nantes, France \\ Email: kevin.nadaud@univ-nantes.fr
}

\author{
Raphaël Gillard and Erwan Fourn \\ Institut d'Électronique et de Télécommunications de Rennes \\ INSA of Rennes \\ Rennes, France \\ Email: raphael.gillard@insa-rennes.fr
}

\begin{abstract}
In this paper we propose a new method of dielectric characterization of high-k thin films based on the measurement of coplanar capacitor inserts between two coplanar waveguide transmission lines. The measurement geometry is deposed on the thin film which is elaborate on an insulating substrate. The thin film permittivity is extracted with the help of a mathematical model describing the capacitance between two conductor plates deposed on a 2-layers substrate. A simple correction is proposed in order to enhance the matching between the model and the full wave simulation. The results of the proposed measurement method are compared to those of a classical characterization technique using parallel plate capacitor geometry.
\end{abstract}

Keywords-Ferroelectric, thin film, characterization, microwave, coplanar waveguide $(\mathrm{CPW})$.

\section{INTRODUCTION}

Ferroelectric thin films are widely studied for their non constant dielectric permittivity which allows realization of electrically tunable components and devices such as microwave filters, reflectarray antennas, resonators or phase shifters. Depending on the component architecture, the films have to be elaborated either on a conducting electrode or an insulating substrate. As thin films synthesis, and hence dielectric properties, strongly depend on the substrate's crystalline structure, it is important to use the same topology for the material characterization and the final component.

MIM (Metal Insulator Metal) topology is commonly used in order to characterize thin films, [1]-[4]. However, at high frequencies or in the case of a high permittivity, propagation phenomena reduce the apparent permittivity. A solution would be patterning smaller electrodes, with the inconvenience to make fabrication and measurement more difficult. Moreover, the fringing field at the metalization edges would becomes more important and should be considered.

Most characterization methods relying on CoPlanar Waveguide (CPW) technology are based on propagation constant or effective permittivity measurements. The obtained accuracy is usually poor because the film permittivity only little contributes to the effective permittivity as the distance between conductors is important compared with the film thickness. The proposed method allows measuring the complex permittivity of a ferroelectric thin film in the microwave range using a coplanar capacitance with a narrow gap.

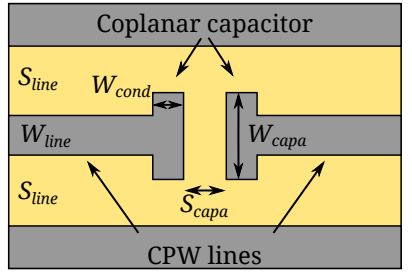

(a)

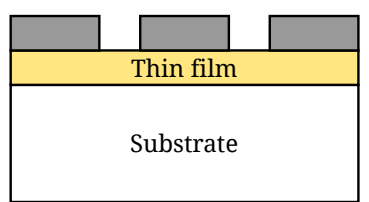

(b)
Figure 1. Proposed topology to extract complex permittivity. In practice, $W_{\text {line }}=30 \mu \mathrm{m}, S_{\text {line }}=150 \mu \mathrm{m}, W_{\text {cond }}=30 \mu \mathrm{m}, W_{\text {capa }}=60$ to $150 \mu \mathrm{m}$ and $S_{\text {capa }}=4$ to $12 \mu \mathrm{m}$. The thin film thickness is $1 \mu \mathrm{m}$.

\section{Measurement TOPOLOGY}

The proposed topology is a coplanar capacitor formed by two plates of width $W_{\text {capa }}$ separated by the distance $S_{\text {capa }}$ and connected to CPW transmission lines, Fig. 1. Lines allow measuring the capacitance without disturbing it and the use of GSG (Ground Signal Ground) probes. Different capacitor geometries are used, the line and gap width of the CPW transmission line, respectively $W_{\text {line }}$ and $S_{\text {line }}$, are kept unchanged allowing to use the same de-embedding set for the measurements. Different widths of plates $W_{\text {capa }}$ are necessary to perform the proposed correction explained later on.

In order to extract permittivity of the thin film, a mathematical model is needed as the capacitance between the two conductors cannot be determined as easily as in a MIM configuration. Gevorgian [5] proposes an equivalent $\pi$ network for a CPW series gap using conformal mapping but assumes an infinitely thick substrate, which cannot be applied here. In [6] a complete model describes the gap discontinuity but extraction of thin film permittivity is not easy. In our case, we only consider the two plates deposed on the film, not the complete discontinuity with the ground. This simplification allows using a more simpler model for computing the capacitance.

We do not choose the model proposed by Gevorgian [7], which takes account of the fringing field at the extremities, because it has a "fitting parameter". The parameter is an integration constant and can be set to any value in theory. In practice, this parameter can match the capacitance given by the model to the simulation's one, but has no physical signification.

Our approach is based on Vendik's model [8] which gives the capacitance between two coplanar plates but without taking 


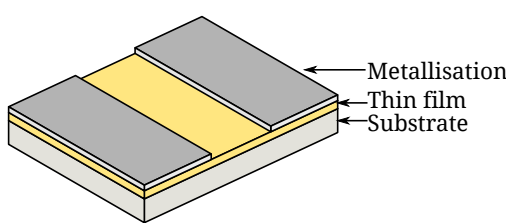

(a)

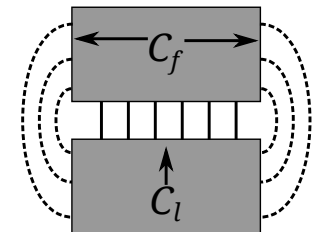

(b)
Figure 2. Topology decribes by Vendik's model. Solid lines represent the linear capacitance $C_{l}$ and dash lines the fringing field capacitance $C_{f}$ at the plates extremities. The model only considers the contribution of the linear capacitance $C_{l}$.

account the fringing field at the extremities of the plates. It only considers the linear contribution $C_{l}$, Fig. 2. This model seems to be less precise but has been chosen because a simple correction can remove the fringing field part from the overall measured or simulated capacitance. To avoid dimension limitations, the complete form given by Vendik is used to compute the capacitance:

$$
\begin{gathered}
C_{\text {Vendik }}=C_{l} \times W_{\text {capa }} \\
C_{l}=\varepsilon_{0}\left[\frac{K\left(k_{a}^{\prime}\right)}{K\left(k_{a}\right)}+\frac{\varepsilon_{f}-\varepsilon_{s}}{2} \frac{K\left(k_{f}^{\prime}\right)}{K\left(k_{f}\right)}+\frac{\varepsilon_{s}-1}{2} \frac{K\left(k_{s}^{\prime}\right)}{K\left(k_{s}\right)}\right] \\
k_{a}=\frac{S_{\text {capa }}}{l}, \quad k_{a}^{\prime}=\sqrt{1-k_{a}} \\
k_{f}=\frac{\tanh \left(\frac{\pi S_{\text {capa }}}{4 h_{f}}\right)}{\tanh \left(\frac{\pi l}{4 h_{f}}\right)}, \quad k_{f}^{\prime}=\sqrt{1-k_{f}} \\
k_{s}=\frac{\tanh \left(\frac{\pi S_{\text {capa }}}{4\left(h_{f}+h_{s}\right)}\right)}{\tanh \left(\frac{\pi l}{4\left(h_{f}+h_{s}\right)}\right)}, \quad k_{s}^{\prime}=\sqrt{1-k_{s}}
\end{gathered}
$$

where $K$ is the complete elliptic integral of the first kind, $\varepsilon_{f}$ and $\varepsilon_{s}$ are respectively the relative permittivity of the ferroelectric thin film and the substrate and $h_{f}, h_{s}$ refer to film thickness and substrate thickness. With $W_{\text {cond }}$ being the length of the plates, we have $l=S_{\text {capa }}+2 W_{\text {cond }}$. The coplanar discontinuity is modeled by a $\pi$ network presented Fig. 3 . In measurement, S-parameters permit to determine the series capacitance $C_{s}$ and the shunt one $C_{p}$ by using the admittance matrix.

$$
\left[\begin{array}{ll}
Y_{11} & Y_{12} \\
Y_{21} & Y_{22}
\end{array}\right]=\left[\begin{array}{cc}
j \omega\left(C_{s}+C_{p}\right) & -j \omega C_{s} \\
-j \omega C_{s} & j \omega\left(C_{s}+C_{p}\right)
\end{array}\right]
$$

Using (6), we can extract the series and the shunt capacitance:

$$
\begin{gathered}
C_{s}=\frac{-Y_{12}}{2 \pi j f} \\
C_{p}=\frac{Y_{11}+Y_{12}}{2 \pi j f}
\end{gathered}
$$

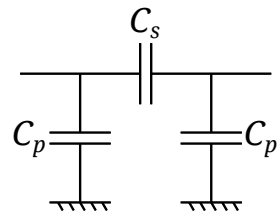

Figure 3. Equivalent $\pi$ network of the proposed topology.

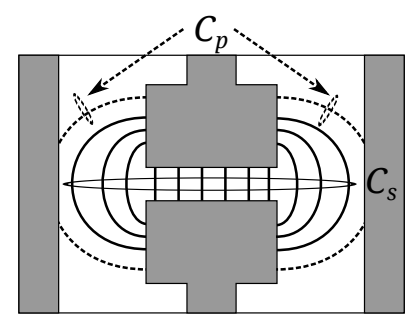

(a)

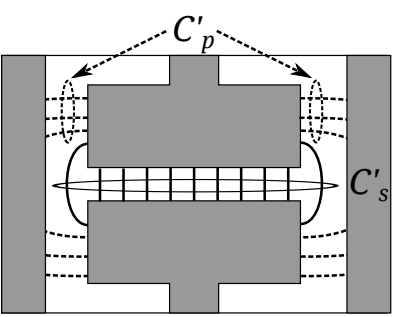

(b)
Figure 4. Solid lines represent the series capacitance $C_{s}$ and dash lines the capacitance with the ground $C_{p}$. For small widths, the most part of the fringing field is between the plates (a). When the width of the plates increase, the ground catchs a part of the fringing field: $C_{s}$ reduces and $C_{p}$ grows (b).

\section{SimUlation OF THE CAPACITOR}

The structure shown in Fig. 1 has been simulated with the commercial software HFSS. Particular attention has been given to mesh generation in order to avoid possible issues resulting from high contrasts of size. In our case, the total simulated substrate surface is $2 \times 2 \mathrm{~mm}^{2}$ and the ferroelectric thin film has a thickness of only $1 \mu \mathrm{m}$. When using the default mesh settings, the results are not consistent. Consequently, a finer mesh has to be enforced in the capacitor region in order to obtain reproducible and conclusive results. Different capacitor geometries (distances between coplanar plates $S_{\text {capa }}$ and width $W_{\text {capa }}$ ) will be presented to compare the model and the simulation on numerous configurations.

\section{A. Without correction}

Fig. 5a shows the result of the simulation and the computed capacitance given by Vendik's model without applying any correction. A considerable difference between the simulation and the model is observed which indicate the contribution of the fringing field cannot be neglected. As a consequence, without correction, the model cannot be used for extracting the thin film permittivity.

\section{B. With correction but without ground effect}

The basic correction assumes that the capacitance $C_{f}$, created by fringing field at the extremities of the plates (Fig. 2b), does not depend on $W_{\text {capa }}$ since $W_{\text {cond }}$ and $S_{\text {capa }}$ stay constant. It also neglects the influence of the ground on the parasitic capacitance. Hence, we can write the series capacitance:

$$
C_{s}=C_{l} \times W_{\text {capa }}+C_{f}
$$

Using a linear regression on the series capacitance $C_{s}$, the $Y$-intercept $C_{f}$ can be extracted and deduced from $C_{s}$. The regression has been made for each distance between the conductors because the fringing field is modified when the distance $S_{\text {capa }}$ changes. The result of the correction is visible in Fig. $5 \mathrm{~b}$. The relative error is considerably lower than in the case without 

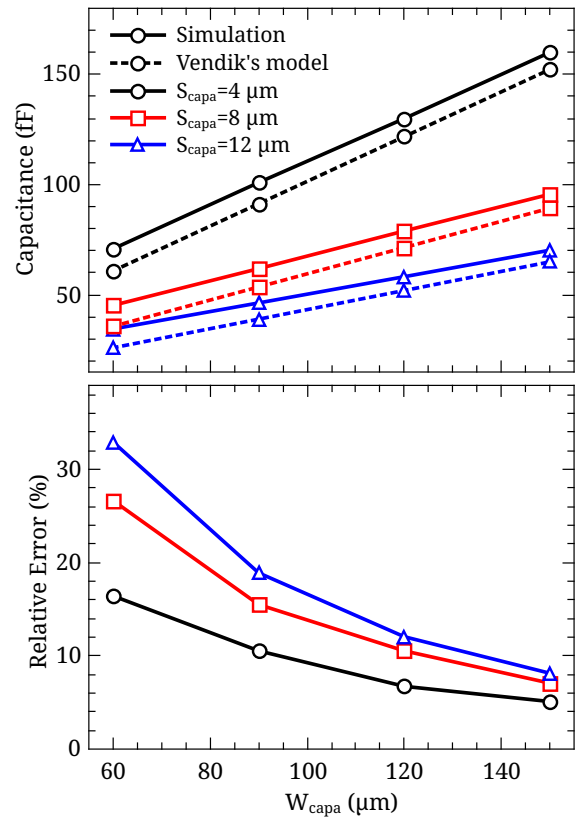

(a)
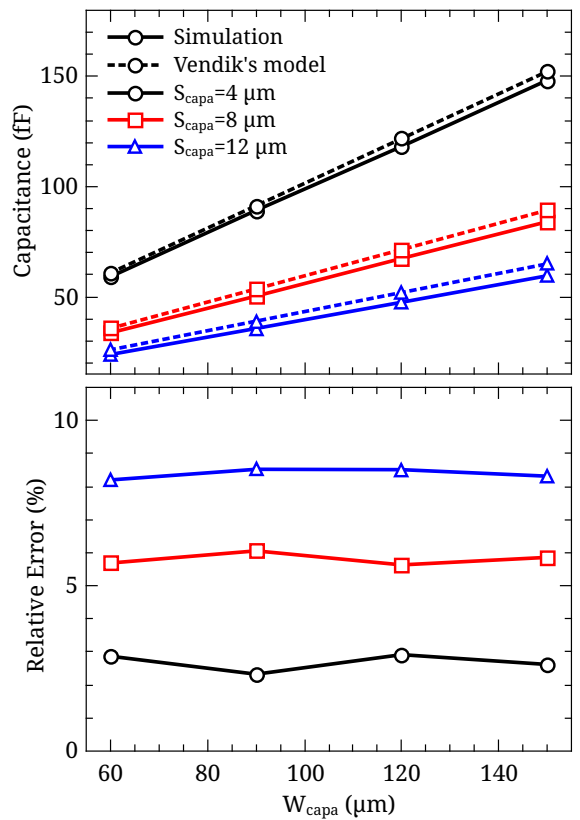

(b)

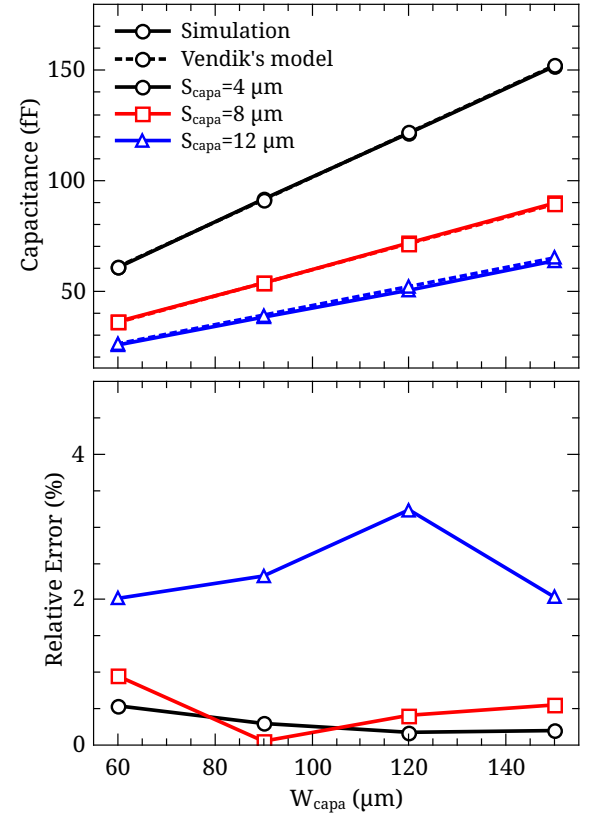

(c)

Figure 5. Comparisons between Vendik's model and HFSS simulations. Without correction (a), with correction but no ground consideration (b), with correction and ground consideration (c).

any correction, especially when the distance between plates $S_{\text {capa }}$ is small. For the smaller distances between plates, the relative error is approximately $3 \%$ and $8 \%$ for the biggest distance. Hence we suppose that the effect of the ground cannot be neglected but has to be taken into account.

\section{With correction and ground consideration}

The elaborate correction consists in taking into account the capacitance between the plates and the ground. While extracting the series capacitance $C_{s}$, from the S-parameters measurements, we also get the ground capacitance $C_{p}$ (eq. 8). Assuming the ground does not disturb too much the fringing field but catches a part of it: when the width $W_{\text {capa }}$ increases, $C_{f}$ reduces and $C_{p}$ grows, cf Fig. 4. We have the sum $C_{f}+\frac{C_{p}}{2}$ constant for each width $W_{\text {capa }}$ and now the linear regression is made on $C_{s}+\frac{C_{p}}{2}$. The result of this correction is shown in Fig. 5c. The mean relative error become around $2.5 \%$ for the largest capacitor gap (less favorable case) and is below $1 \%$ for the smallest gaps, $S_{\text {capa }}$. For experimental characterization of high-k thin film, the capacitor topology that gives the best precision has small distance between the conductor plates and large distance with the ground. In this case, a simple correction allows removing the fringing field at the extremities of the plates and the Vendik's model can be used to extract the thin film permittivity.

\section{EXPERIMENTAL RESULTS}

$\mathrm{Ba}_{0.80} \mathrm{Sr}_{0.20} \mathrm{TiO}_{3}$ (BST) thin films were elaborated on alumina substrates by using a modified sol-gel method developed in [9]. The coplanar capacitor structure consists of a $1 \mu \mathrm{m}$ thick copper layer deposed by sputtering and pattern with photolithography. The dimensions of the capacitor are measured with a $\pm 0.3 \mu \mathrm{m}$ accuracy. The width of the gap $S_{\text {capa }}$

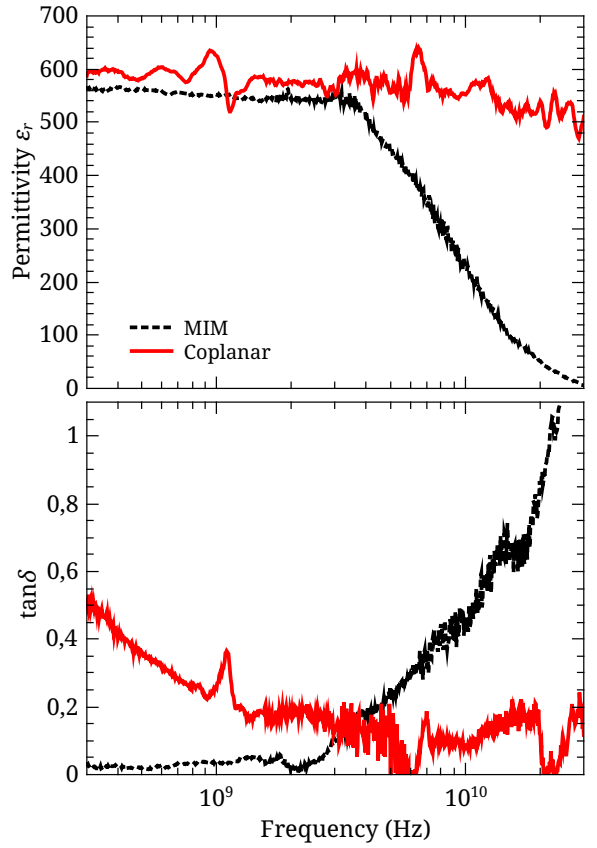

Figure 6. Estimated permittivity and losses in MIM and coplanar topologies. At high frequencies, MIM topology cannot be used because propagation effects reduce apparent permittivity. The coplanar topology can be used at higher frequencies because no propagation effects are present.

is really critical because the capacitance strongly depends on it when it is small. BST is also deposed on platinum coated alumina substrates to have an estimation of the relative permittivity of the film in a MIM topology.

The smallest gap size $S_{\text {capa }}$ realized were $7.5 \mu \mathrm{m}$ which 
corresponds to the intermediate value utilized for the simulations (cf Fig. 5c). The relative error of the permittivity extracted from the S-parameters hence can be estimated to $1 \%$. A TRL (Thru Reflect Line) calibration has been done to remove the effect of the connection lines, probes and cables.

\section{A. Permittivity measurements}

Fig. 6 shows the measured relative permittivity $\varepsilon_{r}$. For the characterization in MIM geometry, an abrupt decrease of the estimated permittivity can be seen at $3 \mathrm{GHz}$. This is due to the propagation effect and indicates the frequency limit of this technique. The results obtained above this frequency are not reliable. In the case of the coplanar capacitor topology, the extracted permittivity corresponds to the one determined in the MIM topology at the left side side of the graph. A continuous behavior of the permittivity evolution is obtained up to approximately $10 \mathrm{GHz}$ and a slight decay can be observed at higher frequencies. The difference of the estimated value of permittivity is probably due of the platinum coating which can modify the dielectric properties of the film deposed above. This shows the importance of characterized the material in the same topology of the final application. In measurement, the shunt capacitance $C_{p}$ has not been extracted because the calibration is not precise enough. Only the basic correction has been made here, which results in a slightly underestimated relative permittivity.

\section{B. Extracted losses}

Fig. 6 also shows the measured dielectric losses. At low frequencies, $\tan \delta$ given by the MIM topology is around 0.02 . At high frequencies, losses are false because the permittivity is not extracted correctly. This indicates again the limitation of the MIM topology. For the coplanar capacitor, losses are overestimated. The reason is that metallic losses are also measured and added to dielectric ones. A solution is to depose thicker metallic layer, to reduce the contribution of metallic losses.

\section{CONClusions}

In this paper we describe a new method for the dielectric characterization of high-k thin films in the microwave range. The proposed method consists in measuring coplanar capacitor inserts into two CPW lines. A basic model and a simple correction are proposed to extract the complex permittivity.
The proposed model is in very good agreement (below $1 \%$ ) with numerical simulation for small gap between plates. In measurement the coplanar characterization involves no propagation phenomena contrary to MIM topology and permittivity can be extracted at much higher frequencies. There is also a good agreement between permittivity extracted with both methods which comfort the validity of the proposed technique. Smaller capacitors gaps will allow better agreement between the reality and model with correction. Performing a better calibration will permit to extract shunt capacitance in order to applied the elaborate correction. Thicker metalization shall reduce the contribution of metallic losses and hence shall make the measurement technique also suitable for the characterization of the material's dielectric losses. Optimizations certainly allow future high-k thin film characterization up to several tenths of GHz.

\section{REFERENCES}

[1] Z. Ma, A. J. Becker, P. Polakos, H. Huggins, J. Pastalan, H. Wu, K. Watts, Y. H. Wong, and P. Mankiewich, "RF measurement technique for characterizing thin dielectric films," IEEE Trans. Electron Devices, vol. 45, pp. 1811-1816, 1998.

[2] M. P. J. Tiggelman, K. Reimann, J. Liu, M. Klee, W. Keur, R. Mauczock, J. Schmitz, and R. J. E. Hueting, "Identifying dielectric and resistive electrode losses in high-density capacitors at radio frequencies," in Proc. IEEE ICMTS, 2008, pp. 190-195.

[3] M. P. J. Tiggelman, "Thin film barium strontium titanate capacitors for tunable RF front-end applications," Ph.D. dissertation, University of Twente, 2009.

[4] W. Chen, K. G. McCarthy, A. Mathewson, M. Copuroglu, S. O’Brien, and R. Winfield, "Capacitance and S-parameter techniques for dielectric characterization with application to high-k PMNT thin-film layers," IEEE Trans. Electron Devices, vol. 59, no. 6, pp. 1723-1729, 2012.

[5] S. Gevorgian, A. Deleniv, T. Martinsson, S. Gal'chenko, P. Linnér, and I. Vendik, "Cad model of a gap in a coplanar waveguide," International Journal of Microwave and Millimeter-Wave Computer-Aided Engineering, vol. 6, no. 5, pp. 369-377, 1996.

[6] A. Deleniv, I. Vendik, and S. Gevorgian, "Modeling gap discontinuity in coplanar waveguide using quasistatic spectral domain method," International Journal of RF and Microwave Computer-Aided Engineering, vol. 10, no. 3, pp. 150-158, 2000.

[7] S. Gevorgian, Ferroelectrics in microwave devices, circuits and systems. Springer, 2009.

[8] O. G. Vendik, S. P. Zubko, and M. A. Nikolskii, "Modeling and calculation of the capacitance of a planar capacitor containing a ferroelectric thin film," Technical Physics, vol. 44, no. 4, pp. 349-355, April 1999.

[9] C. Borderon, D. Averty, R. Seveno, and H. W. Gundel, "Influence of the morphology of barium strontium titanate thin films on the ferroelectric and dielectric properties," Integrated Ferroelectrics, vol. 97, no. 1, pp. 12-19, 2008. 